Typeset with jpsj2.cls $<$ ver.1.0beta $>$

\title{
Theoretical Description of Nearly Discontinuous Transition in Superconductors with Paramagnetic Depairing
}

\author{
Hiroto Adachi ${ }^{*}$, Shigeru KolKegami ${ }^{1}$, and Ryusuke IKeda \\ Department of Physics, Kyoto University, Kyoto 606-8502, Japan \\ ${ }^{1}$ Nanoelectronics Research Institute, AIST Tsukuba Central 2, 305-8568, Japan
}

(Received November 20, 2018)

\begin{abstract}
Based on a theoretical argument and Monte Carlo simulations of a Ginzburg-Landau model derived microscopically, it is argued that, in type-II superconductors where both the paramagnetic and orbital depairings are important, a strong first order transition (FOT) at $H_{c 2}$ expected in the mean field (MF) approximation never occurs in real systems and changes due to the fluctuation into a crossover. The present result explains why a nearly discontinuous crossover at $H_{c 2}$ with no intrinsic hysteresis is observed only in a clean superconducting material with a singlet pairing and a high condensation energy such as $\mathrm{CeCoIn}_{5}$.
\end{abstract}

KEYWORDS: paramagnetic and orbital depairings, upper critical field, CeColn ${ }_{5}$, vortex phase diagram

The phase diagram of a type-II superconductor in nonzero magnetic fields $(H \neq 0)$ has been studied in relation to high $T_{c}$ cuprates in fields of the tesla range and beyond the mean field (MF) approximation completely neglecting the superconducting (SC) fluctuation effect. ${ }^{1,2}$ ) Even in a material with a spin-singlet electron-pairing, the spin (i.e., Pauli paramagnetic) depairing effect can be neglected in relatively low fields, and the transition at $H_{c 2}(T)$-line in the MF approximation for this case is of second order. On the other hand, the phase diagram in cases where both the Pauli paramagnetic and orbital depairings are important were examined so far only within the MF approximation. $^{3,4)}$ Since the MF transition in this case has been expected to become first order at low enough temperatures, ${ }^{3,4)}$ it is valuable to clarify whether this MF scenario is also, as well as in the low field case, ${ }^{1,2)}$ drastically changed by including the SC fluctuation.

In this paper, we first examine the MF approximation of a quasi two-dimensional (2D) superconductor under a field perpendicular to the SC layers $(\boldsymbol{H} \| c)$ by treating the orbital- and spin-depairing effects on an equal footing and obtain a first order transition in the MF approximation (MF-FOT) at $H_{c 2}(T)$ and in high fields. Then, based on a physical picture on the high $H$ fluctuation and our Monte Carlo simulation results on the derived Ginzburg-Landau (GL) model, we argue that any nonvanishing SC fluctuation, as well as in the low $H$ case, ${ }^{1,2)}$ changes this MFFOT into a crossover and point out a consistency between the present theory and experimental results, such as the apparently discontinuous behavior ${ }^{5-8)}$ in high fields with no intrinsic hysteresis seen in $\mathrm{CeCoIn}_{5}$ with unusually large condensation energy and the absence of such a sharp behavior

*ada@scphys.kyoto-u.ac.jp 
in other materials ${ }^{9-11)}$ showing large paramagnetic effects and having lower condensation energies.

Let us first briefly explain our microscopic derivation of a Ginzburg-Landau (GL) functional in the case with spin depairing effect by starting with a quasi 2D BCS hamiltonian. An attractive interaction leading to the $d_{x^{2}-y^{2}}$-pairing state will be assumed. For $\boldsymbol{H} \| c$ case, the following GL model will be derived microscopically for a layered system:

$$
\begin{aligned}
\mathcal{F} & =N(0) \int d^{2} r_{\perp}\left[\sum_{q_{z}} \sum_{N} a_{N}\left(q_{z}^{2}\right)\left|\tilde{\Delta}_{q_{z}}^{(N)}\left(\boldsymbol{r}_{\perp}\right)\right|^{2}\right. \\
& \left.+\sum_{j}\left(\frac{V_{4}}{2}\left|\Delta_{j}^{(0)}\left(\boldsymbol{r}_{\perp}\right)\right|^{4}+\frac{V_{6}}{3}\left|\Delta_{j}^{(0)}\left(\boldsymbol{r}_{\perp}\right)\right|^{6}\right)\right]
\end{aligned}
$$

where $\Delta_{j}^{(N)}\left(\boldsymbol{r}_{\perp}\right)=N_{\text {layer }}^{-1 / 2} \sum_{q_{z}} \tilde{\Delta}_{q_{z}}^{(N)}\left(\boldsymbol{r}_{\perp}\right) e^{\mathrm{i} q_{z} s j}$ denotes the pair-field (SC order parameter) on the $j$-th SC layer projected onto the Nth Landau level (LL), $N(0)$ is a 2D density of state at the Fermi surface, $N_{\text {layer }}$ is the number of SC layers, and $s$ is the layer spacing. Below, the higher LL modes with $N \geq 2$ will be neglected. In eq.(1), the 4 th and 6 th order terms were represented within the lowest LL approximation and in a spatially local form. The neglect of this non-locality is justified because the energy scale associated with the MF transition (i.e., the condensation energy) is much larger than that on a phenomenon arising from this non-locality such as a structural transition between the rhombic and square vortex lattices. Then, assuming, for brevity, a cylindrical Fermi surface and treating effects of the magnetic field quasi-classically, the coefficient $a_{N}\left(q_{z}^{2}\right)$ of eq.(1) in pure case $\left(\tau^{-1}=0\right)$ becomes

$$
\begin{aligned}
a_{N}\left(q_{z}^{2}\right)= & \ln \left(T / T_{c 0}\right)-2 \pi T \sum_{\varepsilon>0}\left(D_{N}(2 \varepsilon)-1 / \varepsilon\right) \\
D_{N}(2 \varepsilon)=2 & \int_{0}^{\infty} d \rho e^{-2 \varepsilon \rho-\left(\frac{\rho}{2 \tau_{H}}\right)^{2}} \mathcal{L}_{N}\left(2\left(\frac{\rho}{2 \tau_{H}}\right)^{2}\right) \\
& \times \cos \left(2 \mu_{0} H \rho\right) \mathcal{J}_{0}\left(2 J \sin \left(\frac{q_{z} s}{2}\right) \rho\right) .
\end{aligned}
$$

where $\varepsilon=2 \pi(n+1 / 2) T$ is a Matsubara frequency, $T_{c 0}$ the transition temperature in $H=0, \tau_{H}=$ $r_{H} / v_{F}, v_{F}$ the Fermi velocity, $r_{H}=\sqrt{\phi_{0} / 2 \pi H}$ the magnetic length, $J$ the interlayer hopping, $\mu_{0} H$ the Zeeman energy, and $\mathcal{L}_{N}$ and $\mathcal{J}_{N}$ are the Nth order Laguerre and Bessel functions, respectively. In the impure case with a finite elastic scattering rate $\tau^{-1}$, the impurity-ladder vertex correction can be neglected in non $s$-wave pairing cases if $\tau_{H} \ll \tau .^{3,12)}$ Then, the corresponding expression to eq. (2) is given by replacing $D_{N}(2 \varepsilon)$ by $D_{N}\left(2 \varepsilon+\tau^{-1}\right)$.

The corresponding calculation of the coefficients $V_{4}$ and $V_{6}$ is more involved and requires multiple numerical integrals. The orbital depairing always introduces the field-induced vortices, which may change drastically the phase diagram in $H \neq 0$, and hence, will be included nonperturbatively in contrast to ref. ${ }^{4)}$ Further, the paramagnetic effect becoming important upon cooling is sensitive to the impurity scattering. Hence, both the two depairing effects and the impurity scattering 


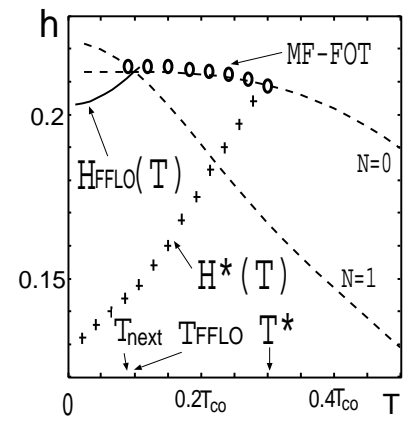

(a)

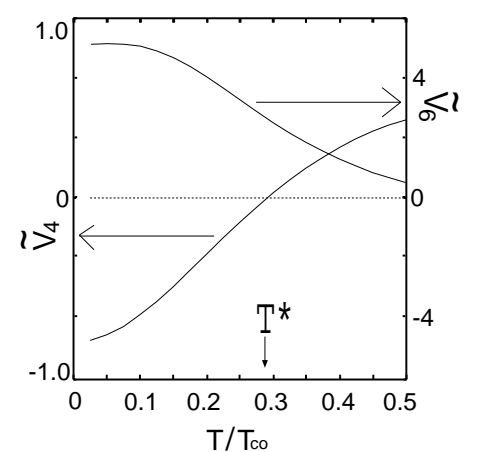

(b)

Fig. 1. (a) Mean field $H$-T phase diagram obtained from computations in high $H$ and low $T$ region. The magnetic field is measured in the reduced variable $h=H / H_{c 2}^{\text {orbit }}$. (b) $T$-dependences of $\tilde{V}_{4}=V_{4} / \tau_{H}^{2}$ and $\tilde{V}_{6}=V_{6} / \tau_{H}^{4}$ computed on $H_{c 2}(T)$. In both (a) and (b), $\left(2 \pi \tau T_{c 0}\right)^{-1}=0.025$ was commonly used. See the text for other details.

should be treated on an equal footing. For this reason, we have examined the coefficients $V_{4}$ and $V_{6}$ numerically by, as well as in eq.(2), applying the parameter ( $\rho$ in eq.(3)) integral representation and neglecting the vertex corrections. Details of their derivation will be described elsewhere. ${ }^{12)}$ Consequently, as shown in Fig.1, the coefficient $V_{4}\left(V_{6}\right)$ defined in $N=0$ becomes negative (positive) in $H>H^{*}(T)$.

In Fig.1 (a), we give a MF phase diagram in high $H$ following from calculations of the GL coefficients mentioned above in a slightly impure $\left(\left(2 \pi \tau T_{c 0}\right)^{-1}=0.025\right)$ case. There, the paramagnetic parameter $\mu_{0} H_{c 2}^{\text {orbit }} / 2 \pi T_{c 0}$ corresponding to the Maki parameter is chosen as 0.8 , where $H_{c 2}^{\text {orbit }}=0.56 \phi_{0} /\left(2 \pi \xi_{0}^{2}\right)$ is the $2 \mathrm{D}$ orbital limiting field in pure case, and $\xi_{0}=v_{F} / 2 \pi T_{c 0}$. The dashed curves express the lines defined by $a_{0}(0)=0$ and $a_{1}(0)=0$, and, in a range where the MF transition is of second order, the higher field portion of these two curves plays the role of the $H_{c 2}(T)$-line. Above the curve denoted by the plus symbols (i.e., $H>H^{*}(T)$ ), however, the coefficient $V_{4}$ in $N=0$ is negative, making the MF transition in $N=0$ a discontinuous one (i.e., FOT) which occurs on the portion of $H_{c 2}(T)$ denoted by the open circles in $T_{\text {next }}<T<T^{*}$. On the other hand, the MF transition in $N=1$ is found to remain continuous, ${ }^{12)}$ although the $N=1$ region ${ }^{13)}$ with $a_{0}(0)>a_{1}(0)$ is quite narrow. We have also estimated a structural transition curve $H_{\mathrm{FFLO}}(T)$ between the ordinary straight vortex solid and a FFLO-like helical vortex solid with a modulation $\Delta_{j}^{(0)} \sim e^{\mathrm{i} q_{z} s j}$ along $\boldsymbol{H}$ as the line where $\partial a_{0}\left(q_{z}^{2}\right) /\left.\partial q_{z}^{2}\right|_{q_{z}=0}=0$. Since $\partial^{2} a_{0}\left(q_{z}^{2}\right) /\left.\partial\left(q_{z}^{2}\right)^{2}\right|_{q_{z}=0}>0$, and the spin depairing inducing this transition is enhanced with increasing $H$ and decreasing $T$, a second order structural transition at $H_{\mathrm{FFLO}}(T)$ decreasing upon cooling is expected at least near $H_{c 2}$ to occur, although the range in which this $N=0$ state is realized seems to be quite narrow in this $\boldsymbol{H} \| c$ case. Actually, in contrast to $T^{*}$, the $H_{\mathrm{FFLO}}(T)$ curve and the $N=1$ vortex state region are very sensitive to the impurity strength, and we find that, for $\left(2 \pi T_{c 0} \tau\right)^{-1}=0.05, T^{*} \simeq 0.23 T_{c 0}$, while the $H_{\mathrm{FFLO}}(T)$ and the $N=1$ region are not present any longer. The main result $T^{*}>T_{\mathrm{FFLO}}$ 
in our MF calculation is satisfied, at least in $\boldsymbol{H} \| c$, irrespective of the impurity strength ${ }^{12)}$ and should be compared with results in ref. ${ }^{4)}$

Now, let us turn to discussing the genuine high $H$ phase diagram of superconductors with paramagnetic depairing. Hereafter, we will focus on the region $H^{*}(T)<H<H_{\mathrm{FFLO}}(T)$ in Fig.1(a) and use the following GL functional on the basis of the MF results shown above:

$$
\begin{gathered}
\frac{\mathcal{F}}{T}=\sum_{j} \int d^{2} r_{\perp}\left\{\alpha\left|\Psi_{j}\right|^{2}+\gamma\left|\Psi_{j}-\Psi_{j+1}\right|^{2}\right. \\
\left.-\frac{|\beta(T)|}{2}\left|\Psi_{j}\right|^{4}+\frac{1}{3}\left|\Psi_{j}\right|^{6}\right\} .
\end{gathered}
$$

Here, $\gamma>0, \alpha \simeq \alpha_{0}\left(H-H_{0}\right) / H_{0}$ with a $T$-dependent constant $\alpha_{0}$, and $H_{0}$ is the field value satisfying $a_{0}(0)=0$. Further, the length and the pair-field were rescaled in the manner $\boldsymbol{r}_{\perp} / r_{H} \rightarrow \boldsymbol{r}_{\perp}$ and $\left(T / V_{6} r_{H}^{2} N(0)\right)^{-1 / 6} \Delta_{j}^{(0)} \rightarrow \Psi_{j}$. Consequently, the $2 \mathrm{D}$ fluctuation strength is measured by $|\beta(T)|^{-1}=\left|V_{4}\right|^{-1}\left(V_{6}^{2} T / N(0) r_{H}^{2}\right)^{1 / 3}$. Any term expressing a vortex pinning effect induced by the electronic impurity scattering was neglected.

To explain why the MF-FOT should not occur in real systems with any nonvanishing fluctuation, it is convenient to start from a description deep in the ordered phase. First, since an inclusion of the orbital depairing requires the presence of field-induced vortices, a low energy excitation in the ordered phase is inevitably an elastic mode of a vortex solid. It is quite clear that the form of the elastic energy and the relation between the phase fluctuation and the vortex displacement ${ }^{14)}$ are unaffected by a difference in the form of nonlinear terms of the GL model. As in the case with no paramagnetic depairing, ${ }^{14)}$ the phase fluctuation is marginally relevant even in $3 \mathrm{D}$ case, and the main rigidity of the phase fluctuation is the shear modulus of the underlying vortex solid. Hence, if the melting of the vortex solid occurs at $H_{m}$ below $H_{c 2}$, the vortex liquid just above $H_{m}$ with vanishing shear modulus has no long range orders and hence, should be continuously connected with the normal phase above $H_{c 2} \cdot{ }^{2}$ In this case, the MF-FOT at $H_{c 2}$ should not occur. Further, to understand this from another point of view, let us note that the quasi 2D SC order parameter in the lowest LL has the form ${ }^{16)}$

$$
\Psi(u, z)=\mathcal{A}(z) e^{-y^{2} /\left(2 r_{H}^{2}\right)} \prod_{i=0}^{N_{s}-1}\left(u-u_{i}(z)\right),
$$

where $u=x+\mathrm{i} y, z=j s, u_{i}(z)$ is the complex coordinate perpendicular to $\boldsymbol{H}$ of the $i$-th vortex, and a Landau gauge was assumed for the external gauge field. Since the vortex positions are highly disordered above $H_{m}$, the fluctuation effect above $H_{m}$ is essentially described only by the amplitude $\mathcal{A}(z)$. However, $\mathcal{A}(z)$ depends only on $z$ irrespective of the form of nonlinear terms in the GL model. That is, since the amplitude fluctuation itself has a reduced dimensionality and is 1D-like in 3D systems $^{2,15)}$ even in the present case, the amplitude fluctuation plays a role of pushing the vortex lattice freezing field down to a lower field, and the MF-FOT should not be realized in real 3D systems. 


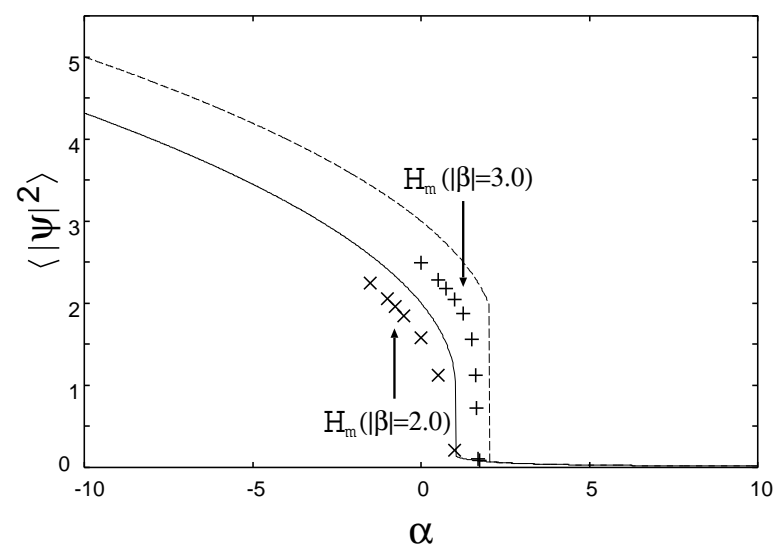

Fig. 2. Numerical data (symbols) of $\left\langle|\Psi|^{2}\right\rangle$ in the case composed of four layers. The corresponding results (curves) in the Hartree approximation ${ }^{15)}$ are also given. The cross symbols and solid curve (plus symbols and dotted curve) are results in $|\beta|=2.0$ (3.0). In $|\beta|=3.0$, the $H_{c 2}(T)$ corresponds to $\alpha=1.7$ and lies above $H_{m}$. The used parameter values are given in the text on the final page.

Bearing such a theoretical prospect in mind, we have performed Monte Carlo simulations on the model of eq.(4), and its 2D version using material parameter values useful for comparisons with observations in $\mathrm{CeCoIn}_{5}$. Our simulation method follows that of ref. ${ }^{17)}$ under the lateral boundary condition $L_{x} / L_{y}=2 N_{x} / \sqrt{3} N_{y}$ with $\left(N_{x}, N_{y}\right)=(6,6)$ and a periodic boundary condition across the layers. Hereafter, the key quantities in the present case within $N=0$ are $-\left\langle|\Psi|^{2}\right\rangle$ and the structure factor defined ${ }^{15)}$ as the correlation function of $\left|\Psi\left(\boldsymbol{r}_{\perp}\right)\right|^{2}$. The former is equivalent to the magnetization or the fluctuation entropy, while the latter is a measure of the vortex positional order.
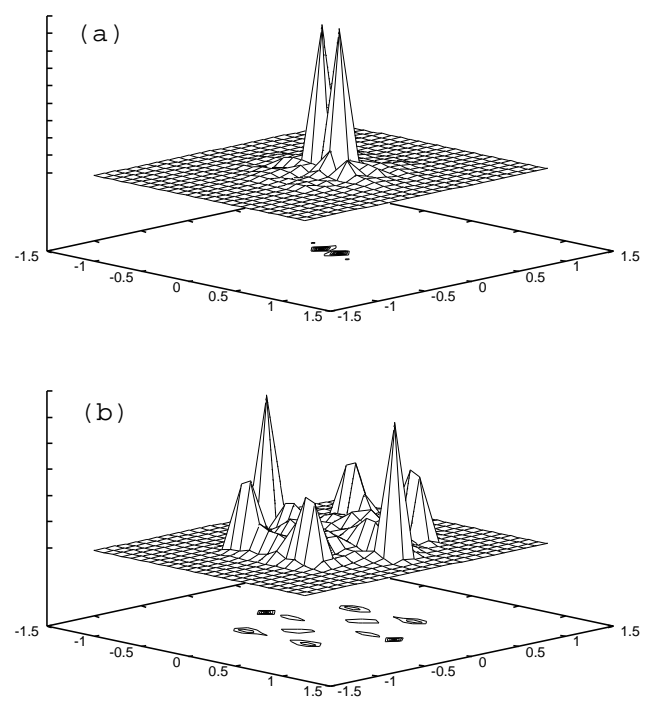

Fig. 3. Structure factor defined from the correlation function of $|\Psi(\boldsymbol{r})|^{2}$ for $|\beta|=3.0$ at (a) $\alpha=1.65$,(b) $\alpha=1.25$.

Computed results of $\alpha$ dependences of $\left\langle|\Psi|^{2}\right\rangle$ in a layered system consisting of four layers are 
shown in Fig.2 for $|\beta|=2.0$ and 3.0. Clearly, the nearly discontinuous behavior at $H_{c 2}$ is smeared out as the fluctuation is enhanced (i.e., $|\beta|$ is reduced). For comparison, the corresponding results in the Hartree approximation ${ }^{15)}$ are also drawn as the solid and dashed curves. Note that a change of $\left\langle|\Psi|^{2}\right\rangle$ at $H_{c 2}$ is extremely steep even in this approximation where no transition occurs. ${ }^{15)}$ Clearly, the presence of a genuine FOT cannot be argued merely through a steep growth of $\left\langle|\Psi|^{2}\right\rangle$ in a real system with weak SC fluctuation. Next, let us examine whether the melting transition point coincides or not with the MF transition field $H_{c 2}(T)$. The melting transition is widely believed to be a weak FOT, and this should be found ${ }^{17)}$ in Monte Carlo simulations as a tiny discontinuity in thermodynamic quantities. Unfortunately, due primarily to numerical difficulties, our simulation is restricted to too small systems to observe such a discontinuity. For our purpose, however, it is sufficient to estimate the melting temperature by finding where the Bragg peaks of the vortex lattice disappear. Fig.3 shows snapshots of the structure factor on $|\Psi|^{2}$ when $|\beta|=3.0$. No vortex positional ordering is seen at $H_{c 2}$. As indicated in Fig.2, nearly sharp Bragg peaks appear at $H_{m}$ below $H_{c 2}$, while most of the entropy have been lost rather near $H_{c 2}$ above it. The two field (or temperature) scales, one characterizing the steep growth of $\left\langle|\Psi|^{2}\right\rangle$ and another corresponding to the sudden growth of vortex positional ordering, are clearly distinguished.

Quite similar results to those in Fig.2 and 3 were also obtained in 2D case where the system size dependence of $H_{m}$ is more easily examined. As expected from a commensurability between a MF solution and the lateral periodic boundary condition, it was verified through the data in system sizes $(6,4)$ and $(8,6)$ with $L_{x} / L_{y}=\sqrt{3} N_{x} / 2 N_{y}$ that $H_{m}$ is lowered with increasing the system size. This means that the relation $H_{m}<H_{c 2}$ found above holds in simulations for larger sizes.

Recently, a large apparent discontinuity was observed in transport and thermodynamic data ${ }^{6-8)}$ in a heavy fermion material $\mathrm{CeCoIn}_{5}$ in high fields, where a strong paramagnetic depairing is expected even in $\boldsymbol{H} \| c,{ }^{6,18)}$ and was identified with the MF-FOT. A hysteresis seen in the magnetization data was interpreted as being due to a genuine FOT at $H_{c 2}$. However, the specific heat in $\boldsymbol{H} \| c^{8)}$ and resistivity data ${ }^{7)}$ have shown no measurable hysteresis in contrast to observations ${ }^{19)}$ in systems with no orbital depairing, although a stronger genuine FOT should be accompanied by a larger hysteresis reflecting a larger condensation energy. Further, a magnetic hysteresis in type-II superconductors is usually a consequence of vortex pinning. ${ }^{20)}$ Actually, a magnetization measurement in $\mathrm{CeCo}_{1-x} \mathrm{Rh}_{x} \mathrm{In}_{5}$ has shown ${ }^{9)}$ that the discontinuity of magnetization disappears with increasing the Rh-substitution, while the hysteresis remains unaffected, implying that the observed $^{6,7)}$ magnetic hysteresis is not a direct consequence of an intrinsic SC transition. Further, the Rh-substitution has resulted in a decrease of condensation energy ${ }^{9)}$ which is usually equivalent to an enhancement of SC fluctuation. Together with the absence of a nearly discontinuous behavior in other systems ${ }^{10,11)}$ suggestive of a large spin depairing and with strong fluctuation, these observations in $\mathrm{CeCoIn}_{5}$ are consistent with the absence of a genuine FOT stressed in this paper. 
Theoretical Description of Nearly Discontinuous Transition in Superconductors with Paramagnetic Depairing 7

Bearing these observations in Ce-compounds in mind, we have chosen parameter values in Fig.2 and 3 as follows. The $N(0)$-value was taken from the heat capacity data in $H=0 .{ }^{18)}$ The Maki parameter value used in Fig. 1 leading to $T^{*} / T_{c 0} \simeq 0.37$ was chosen again because the apparent FOT behavior is observed in $\left.T<0.3 T_{c 0}{ }^{8}{ }^{8}\right)$ Further, we have assumed, for brevity, $T / T_{c 0}=0.1$ and $\gamma=0.25$ (see eq.(4)). Then, the $|\beta|$-value is located in the range between 2.0 and 3.0 used in our computation, and we have $\alpha_{0}\left(\propto\left(N(0) T_{c 0} / T\right)^{2 / 3}\right) \simeq 50$. This $\alpha_{0}$ value suggests that, on a linear scale of the magnetic field of the order of $H_{c 2}(T=0)$, even the broader simulation curve in $|\beta|=2$ in Fig.2 cannot be distinguished from a strictly discontinuous jump. Further, according to our MF calculation the (apparent) magnetization jump value at $T=50(\mathrm{mK})$ is roughly estimated as $20(\mathrm{G})$ which is comparable with the measured one $\left.(\sim 30(\mathrm{G})) .{ }^{6}\right)$

In conclusion, we have given convincing results implying that a MF-FOT at $H_{c 2}$ in superconductors with field-induced vortices (i.e., orbital depairing) should not occur as a genuine FOT in real systems with any nonvanishing fluctuation effect. Typically, this situation is realized in materials with a singlet pairing and with an enhanced paramagnetic depairing. Assuming a quasi 2D material with the $d_{x^{2}-y^{2}}$-pairing state in $\boldsymbol{H} \| c$, we have performed numerical simulations under a condition presumably compatible with high field $\mathrm{CeCoIn}_{5}$ data suggesting a strong discontinuous transition at $H_{c 2}$. The absence of a genuine FOT at $H_{c 2}$ is consistent with the absence of hysteresis accompanying this discontinuous behavior in $\mathrm{CeCoIn}_{5}$ and unrelated to the vortex pinning. The obtained MF phase diagrams seem to be roughly consistent with the observations in $\mathrm{CeCoIn}_{5}$, including recent data ${ }^{21)}$ suggestive of a structural transition possibly to an FFLO-like vortex solid, if taking account of a nonvanishing impurity concentration. The issues in $\boldsymbol{H} \perp c$ will be considered elsewhere.

After writing an earlier version of the manuscript, additional simulations at lower $T / T_{c 0}(<0.1)$ have been performed, and a small hysteresis in the almost discontinuous behaviors was found to occur in this case. However, since the GL coefficients (i.e., $V_{4}$ and $V_{6}$ in eq.(1)) remain unchanged, this hysteresis is not due to a genuine FOT but a phenomenon appearing on measurable Monte Carlo steps (i.e. time scales) as a reflection of incomplete relaxation in simulations ${ }^{12}$ ) which are inevitably in non-equilibrium. The tiny hysteresis appeared at lower $T$ but above $T_{\mathrm{FFLO}}$ in $\mathrm{CeCoIn}_{5}$ only in $\boldsymbol{H} \perp c^{21)}$ is likely to have a similar origin to this numerical one practically occurring in cases with sufficiently weak fluctuations.

We thank T. Sakakibara, Y. Matsuda, K.Izawa, T. Tayama, R. Movshovich, and K. Machida for stimulative discussions. Part of the numerical computation in this work has been carried out at the Supercomputer Center, Institute for Solid State Physics, University of Tokyo, and at the Yukawa Institute Computer Facility in Kyoto University.

1) E. H. Brandt: Rep. Prog. Phys. 58 (1995) 1465. 
2) R. Ikeda: J. Phys. Soc. Jpn. 70 (2001) 219.

3) V. P. Mineev: Phil. Mag. B 80 (2000) 307.

4) M. Houzet and A. Buzdin: Phys. Rev. B 63 (2001) 184521.

5) K. Izawa et al.: Phys. Rev. Lett. 87 (2001) 057002.

6) T. Tayama et al.: Phys. Rev. B 65 (2002) 180504.

7) T. P. Murphy et al.: Phys. Rev. B 65 (2002) 100514(R).

8) A. Bianchi et al.: Phys. Rev. Lett. 89 (2002) 137002.

9) A. Harita et al.: in Autumn Meeting of the Physical Society of Japan (2002).

10) T. Shibauchi et al.: Phys. Rev. Lett. 86 (2001) 5763.

11) T. J. Lee et al:: Phys. Rev. Lett. 78 (1997) 3555.

12) H. Adachi and R. Ikeda: in preparation.

13) H. Shimahara and D. Rainer: J. Phys. Soc. Jpn. 66 (1997) 3591.

14) R. Ikeda et al.: J. Phys. Soc. Jpn. 61 (1992) 254.

15) R. Ikeda et al.: J. Phys. Soc. Jpn. 59 (1990) 1397.

16) Z. Tesanovic and L. Xing: Phys. Rev. Lett. 67 (1992) 2729.

17) Y. Kato and N. Nagaosa: Phys. Rev. B 48 (1993) 7383.

18) S. Ikeda et al.: J. Phys. Soc. Jpn. 70 (2001) 2248.

19) T. Suzuki et al.: J. Phys. Soc. Jpn. 69 (2000) 1462.

20) A. A. Gapud et al.: Phys. Rev. B 67 (2003) 104516.

21) A. Bianchi et al.: cond-mat/0304420. 